\title{
IMPACTO DAS CAPINAS MECÂNICA E QUÍMICA DO SUB-BOSQUE DE Eucalyptus grandis SOBRE A COMUNIDADE DE FORMIGAS

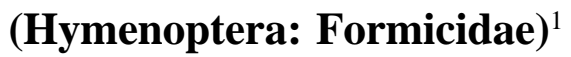

\author{
Lucimeire de Souza Ramos², Ronald Zanetti ${ }^{3}$, Cidália Gabriela Santos Marinho ${ }^{4}$, Jacques Hubert Charles Delabie²; \\ Marcelo Nivert Schlindwein ${ }^{5}$ Roosevelt de Paula Almado ${ }^{6}$
}

\begin{abstract}
RESUMO - O efeito das capinas mecânica e química do sub-bosque em plantações de eucaliptos e as conseqüências desses tratamentos sobre a comunidade de formigas foram avaliados no município de Bom Despacho, Minas Gerais, Brasil. As formigas foram coletadas com o extrator de Winkler. Coletou-se um total de 86 espécies, pertencentes a seis subfamílias. Oito dias após as capinas, o número de espécies reduziu-se de um quarto para os dois tipos de capina. Sessenta dias após, o número de espécies tendeu a retornar ao estágio inicial, verificando-se que a eliminação do sub-bosque causa efeito deletério imediato, de igual intensidade e de pouca duração sobre a comunidade de formigas. As razões das variações observadas serão discutidas.
\end{abstract}

Palavras-chave: Formicidae, eucalipto, manejo, diversidade e serapilheira.

\section{IMPACT OF MECHANICAL AND CHEMICAL WEEDINGS OF EUCALYPTS GRANDIS UNDERGROWTH ON AN ANT COMMUNITY (HYMENOPTERA: FORMICIDAE)}

\begin{abstract}
The effect of mechanical and chemical undergrowth weedings on an ant community was tested in eucalypts plantations and the consequences of such treatments were evaluated at Bom Despacho, Minas Gerais State, Brazil. The ants were collected by applying the Winkler trap method. Eighty-six species of six sub-families were found. Eight days after the clearings, the number of species dropped to 1/4 in both weedings systems. Sixty days later, the number of species tended to return to the initial level, showing that undergrowth elimination caused an immediate depressive effect on the ant community of similar intensity and over a short time. The reasons of the variations observed are discussed.
\end{abstract}

Key words: $\quad$ Formicidae, eucalypts, management, diversity, litter.

\section{INTRODUÇÃO}

O plantio de Eucalyptus spp. (Myrtales, Myrtaceae) ocupa grandes áreas do Estado de Minas Gerais. A partir do momento que se reconheceu o valor de sua madeira, houve a preocupação de explorar mais racionalmente as áreas plantadas, enquanto os problemas ligados à preservação do ambiente e as conseqüências do plantio somente foram enfocados mais tarde (Golfari, 1975, Lima, 1993), visto que muitos impactos sobre a biodiversidade têm sido detectados (Paula, 1997; Peck et al., 1998; Louzada et al., 2000; Andersen et al., 2002).

Recebido para publicação em 14.11.2002 e aceito para publicação em 17.2.2004.

2 U.P.A. Laboratório de Mirmecologia. Convênio UESC/CEPLAC, Caixa Postal 7, 45660-000 Itabuna-BA, Brasil, <ramosls@cepec.gov.br>, <delabie@ cepec.gov.br>. ${ }^{3}$ Departamento de Entomologia da Universidade Federal de Lavras - UFLA, 37200-000 Lavras-MG, Brasil, <zanetti@ufla.br>. ${ }^{4}$ Departamento de Biologia Animal/Entomologia Agrícola da Universidade Federal de Viçosa - UFV, 36570-000 Viçosa-MG, Brasil, <marinho@insecta.ufv.br>. ${ }^{5}$ Rua Borba Gato, 471 Jd Centenário, $13564-$ 100 São Carlos-SP, Brasil, <mnivert@uol.com.br>. 6 CAF Santa Bárbara Ltda., Av. Carandaí, 1115, 30130-915 Belo HorizonteMG, Brasil, <ralmado@caf.ind.br. 
Para manutenção das áreas de eucalipto utilizam-se técnicas silviculturais como a capina química (herbicida) ou a mecânica (roçadeira), que servem para retirada de plantas invasoras e para facilitar a localização das colônias de formigas-cortadeiras dentro do eucaliptal. Paula (1997) relatou que o uso deste tipo de tratamento é desnecessário, pois traz perdas ecológicas, uma vez que reduz a biodiversidade de herbívoros e organismos decompositores e ainda aumenta a ocorrência de pragas devido à redução de inimigos naturais.

A falta de uma cobertura vegetal diversificada no solo, devido a capinas repetidas, favorece as formigas Attini. Pesquisas indicam que o desenvolvimento do subbosque reduz a quantidade média de novos sauveiros a cada ano (Almeida et al., 1983b). Em tais casos, a presença do sub-bosque pode dificultar o pouso e a instalação desses insetos, uma vez que eles tendem a pousar somente em locais limpos e de solo desnudo (Almeida et al., 1983a).

Uma excelente função das formigas em áreas antropizadas é que elas podem ser utilizadas como indicadores biológicos, ferramenta de inestimável utilidade. Elas podem indicar o grau de perturbação do ambiente ou permitem avaliar a dinâmica de recuperação de uma área após um distúrbio (Peck et al., 1998; Louzada et al., 2000; Andersen et al., 2002; Ramos et al., 2003). Com a presença desses insetos é possível avaliar o impacto do desmatamento, utilizando características das comunidades para indicar o efeito desse processo sobre a fauna original, determinar quais ambientes estão sendo manejados de forma adequada à preservação da biodiversidade local e quais os tipos de tratamentos afetam a diversidade do inseto estudado e, a partir de dados avaliados, propor técnicas de manejo florestal que minimizem perdas da diversidade.

O presente estudo foi realizado com o objetivo de avaliar o efeito em função do tempo da capina química e mecânica sobre a diversidade de formigas em eucaliptais.

\section{MATERIAL E MÉTODOS}

O estudo foi conduzido no período de junho a setembro de 2000, em áreas pertencentes à CAF Santa Bárbara Ltda., localizadas no município de Bom Despacho (4522' W; 1941'S), Estado de Minas Gerais, Brasil, cuja altitude média é de $695 \mathrm{~m}$. As amostragens dos formicídeos foram obtidas em duas áreas de monocultura de eucalipto com sub-bosque desenvolvido, tendo na primeira sido realizada a capina mecânica com roçadeira acoplada a um trator. Essa área possui 52 ha de plantação de Eucalyptus grandis W. Hill ex Maiden. A segunda área possui 38,70 ha, ocupada também por Eucalyptus grandis, em que foi aplicado o herbicida glifosato (4 litros de calda/ha), em uma dosagem de $160 \mathrm{~g}$ i.g./ha, também com o auxílio de um trator acoplado a um pulverizador.

As coletas foram efetuadas em três ocasiões: a primeira antes do tratamento (capina) e a segunda e a terceira aos 8 e 60 dias após as capinas, respectivamente. Em cada tratamento foram retiradas 50 amostras aleatórias, utilizando o método do extrator de Winkler, a uma distância mínima de $200 \mathrm{~m}$ dos limites da área, para evitar o efeito de borda. Para garantir a independência das amostras, um intervalo de $50 \mathrm{~m}$ foi respeitado entre as amostras consecutivas (Delabie, 1999; Bestelmeyer et al., 2000). Recentemente, Faria et al. (2001), em uma amostragem de Formicidae com pit-fall, relataram que $15 \mathrm{~m}$ são suficientes para garantir essa independência.

Os formicídeos coletados foram montados e identificados, utilizando-se a coleção de referência do Laboratório de Mirmecologia da CEPLAC/CEPEC e a nomenclatura de Bolton (1995).

Os resultados foram tabelados na forma de matriz e a riqueza esperada (Chao2) e o índice de diversidade de Shannon-Weaver $(H)$ foram calculados com o uso do programa EstimateS (Statistical Estimation of Species Richness and Shared Species from Samples) (Colwell, 1997).

Considerando que no decorrer da análise a maioria das espécies se mostrou rara, foi estudado em detalhe o caso das espécies que apareceram pelo menos 20 vezes em cada área.

\section{RESULTADOS E DISCUSSÃO}

Nos eucaliptais, coletou-se um total de 86 espécies de formigas, distribuídas em 34 gêneros (Quadro 1). Observou-se que não houve diferenças significativas entre as áreas estudadas 8 e 60 dias após as capinas mecânica e química (ANOVA, $p>0,05$ ). Quanto ao efeito das capinas em função do tempo, ambas afetaram o número de espécies de formigas encontradas por amostra (Quadro 2). Aos oito dias, foram observadas reduções da frequiência relacionada a esse tipo de manejo, constatando-se rápido efeito negativo sobre a diversidade 
estudada (ANOVA, p > 0,05). Provavelmente, a redução do número de formigas logo após as aplicações do herbicida e da capina com a roçadeira deveu-se ao distúrbio da eliminação do sub-bosque. No final do período de observação, a comunidade pareceu bastante resiliente.

A riqueza observada e esperada e o índice de diversidade são sistematicamente maiores antes do tratamento
(Quadro 3). Os resultados mostraram que aos oito dias após a realização dos tratamentos os valores de riqueza observada, riqueza estimada e índice de diversidade diminuíram, enquanto aos 60 dias esses valores tornaramse similares aos inicias, indicando não ter havido um grande distúrbio por parte dos tratamentos sobre a diversidade de formigas (Quadro 3).

Quadro 1 - Formicidae coletadas em áreas de eucaliptais aos 0, 8 e 60 dias após as capinas química e mecânica. Bom Despacho-MG/Brasil. J unho a setembro de 2000

Table 1 - Formicidae collected in Eucalyptus plantations 0, 8 and 60 days after chemical and mechanical weedings. Bom Despacho, MG, Brazil. J une to September, 2000

\begin{tabular}{|c|c|c|c|c|c|c|}
\hline \multirow{3}{*}{ Espécie } & \multicolumn{3}{|c|}{ Capina Mecânica } & \multicolumn{3}{|c|}{ Capina Química } \\
\hline & Tempo 0 dias & Tempo 8 dias & Tempo 60 dias & Tempo 0 dias & Tempo 8 dias & Tempo 60 dias \\
\hline & \multicolumn{6}{|c|}{ Número de observações } \\
\hline Acromyrmex balzani & 2 & 0 & 0 & 0 & 0 & 0 \\
\hline Acromyrmex subterraneus brunneus & 0 & 0 & 0 & 0 & 0 & 1 \\
\hline Anochetus diegensis & 4 & 8 & 15 & 7 & 3 & 11 \\
\hline Apterostigma sp.1 & 2 & 2 & 0 & 0 & 0 & 2 \\
\hline Apterostigma sp. 3 & 3 & 3 & 3 & 4 & 1 & 0 \\
\hline Atta sexdens & 2 & 0 & 0 & 0 & 0 & 0 \\
\hline Brachymyrmex sp.1 & 12 & 9 & 23 & 14 & 11 & 11 \\
\hline Brachymyrmex sp.4 & 0 & 0 & 0 & 1 & 0 & 0 \\
\hline Camponotus rufipes & 8 & 4 & 9 & 7 & 6 & 12 \\
\hline Camponotus crassus & 11 & 3 & 2 & 7 & 0 & 5 \\
\hline Camponotus melanoticus & 0 & 0 & 0 & 1 & 0 & 0 \\
\hline Camponotus trapezoideus & 1 & 4 & 3 & 9 & 2 & 6 \\
\hline Camponotus renggeri & 1 & 0 & 0 & 0 & 0 & 0 \\
\hline Cardiocondyla wrougthoni & 0 & 0 & 0 & 0 & 1 & 0 \\
\hline Cephalotes pusillus & 1 & 0 & 0 & 1 & 0 & 1 \\
\hline Crematogaster sp.1 & 2 & 0 & 0 & 1 & 0 & 0 \\
\hline Crematogaster sp. 3 & 0 & 1 & 0 & 1 & 1 & 0 \\
\hline Crematogaster sp.4 & 8 & 2 & 5 & 0 & 2 & 10 \\
\hline Crematogaster sp.5 & 1 & 0 & 0 & 0 & 0 & 2 \\
\hline Crematogaster sp.6 & 0 & 1 & 0 & 0 & 0 & 0 \\
\hline Cyphomyrmex transversus & 2 & 2 & 4 & 3 & 0 & 6 \\
\hline Ectatomma edentatum & 2 & 2 & 1 & 3 & 1 & 2 \\
\hline Ectatomma permagnum & 3 & 0 & 1 & 1 & 0 & 1 \\
\hline Ectatomma brunneum & 2 & 0 & 0 & 4 & 2 & 1 \\
\hline Ectatomma planidens & 2 & 0 & 0 & 3 & 0 & 1 \\
\hline Ectatomma tuberculatum & 0 & 0 & 0 & 0 & 0 & 1 \\
\hline Gnamptogenys sulcata & 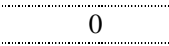 & 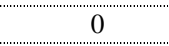 & 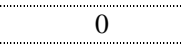 & 0 & 0 & 1 \\
\hline Hypoponera sp.1 & 1 & 1 & 4 & 10 & 5 & 3 \\
\hline Hypoponera sp.2 & 1 & 1 & 1 & 4 & 4 & 7 \\
\hline Hypoponera sp.3 & 0 & 0 & 1 & 2 & 0 & 0 \\
\hline Hypoponera sp.7 & 0 & 0 & 0 & 3 & 1 & 1 \\
\hline Hypoponera sp.4 & 0 & 1 & 0 & 4 & 2 & 0 \\
\hline Hуроропеra foreli & 0 & 0 & 2 & 3 & 1 & 1 \\
\hline Labidus coecus & 2 & 0 & 0 & 1 & 0 & 1 \\
\hline Labidus predator & 0 & 0 & 0 & 1 & 0 & 0 \\
\hline
\end{tabular}

Continua...

Continued... 
Quadro 1, cont.

Table 1, cont.

\begin{tabular}{|c|c|c|c|c|c|c|}
\hline \multirow{3}{*}{ Espécie } & \multicolumn{3}{|c|}{ Capina Mecânica } & \multicolumn{3}{|c|}{ Capina Química } \\
\hline & Tempo 0 dias & Tempo 8 dias & Tempo 60 dias & Tempo 0 dias & Tempo 8 dias & Tempo 60 dias \\
\hline & \multicolumn{6}{|c|}{ Número de observações } \\
\hline Linepithema humile & 0 & 0 & 2 & 0 & 0 & 0 \\
\hline Megalomyrmex sp.1 & 0 & 2 & 0 & 0 & 1 & 0 \\
\hline Mycetarotes acutus & 0 & 0 & 0 & 0 & 1 & 0 \\
\hline Mycocepurus smithi & 3 & 3 & 0 & 5 & 4 & 4 \\
\hline Mycocepurus goeldi & 29 & 23 & 15 & 22 & 19 & 30 \\
\hline Myrmicocrypta foreli & 0 & 0 & 0 & 1 & 0 & 0 \\
\hline Octostruma jheringhi & 2 & 0 & 2 & 5 & 0 & 3 \\
\hline Odontomachus brunneus & 0 & 0 & 0 & 1 & 0 & 1 \\
\hline Odontomachus bauri & 0 & 0 & 0 & 2 & 1 & 1 \\
\hline Odontomachus chelifer & 0 & 0 & 2 & 2 & 0 & 0 \\
\hline Odontomachus meinert & 1 & 0 & 1 & 6 & 3 & 4 \\
\hline Oligomyrmex sp.1 & 0 & 0 & 0 & 0 & 1 & 0 \\
\hline Pachycondyla harpax & 1 & 2 & 1 & 0 & 0 & 0 \\
\hline Pachycondyla gilbert & 0 & 1 & 0 & 0 & 2 & 0 \\
\hline Paratrechina sp.1 & 0 & 0 & 0 & 0 & 3 & 0 \\
\hline Paratrechina sp.3 & 0 & 1 & 0 & 0 & 0 & 0 \\
\hline Pheidole sp.1 & 8 & 4 & 3 & 0 & 7 & 14 \\
\hline Pheidole sp.2 & 2 & 1 & 2 & 5 & 0 & 3 \\
\hline Pheidole sp.3 & 0 & 0 & 4 & 2 & 1 & 5 \\
\hline Pheidole sp.5 & 1 & 0 & 0 & 1 & 0 & 0 \\
\hline Pheidole sp.6 & 0 & 0 & 0 & 2 & 0 & 2 \\
\hline Pheidole sp.7 & 4 & 0 & 0 & 5 & 5 & 0 \\
\hline Pheidole sp.8 & 3 & 1 & 0 & 2 & 0 & 0 \\
\hline Pheidole sp.9 & 0 & 3 & 18 & 3 & 12 & 23 \\
\hline Pheidole sp.11 & 0 & 2 & 3 & 0 & 5 & 3 \\
\hline Pheidole fallax & 4 & 3 & 12 & 6 & 14 & 24 \\
\hline Pogonomyrmex abdominalis & 1 & 0 & 0 & 0 & 0 & 0 \\
\hline Pseudomyrmex simplex & 0 & 0 & 0 & 1 & 0 & 0 \\
\hline Pseudomyrmex tenuis & 1 & 0 & 0 & 1 & 0 & 0 \\
\hline Pseudomyrmex termitarius & 0 & 1 & 0 & 0 & 0 & 0 \\
\hline Pyramica eggersi & 2 & 5 & 12 & 13 & 8 & 21 \\
\hline Pyramica denticulata & 0 & 0 & 3 & 2 & 0 & 5 \\
\hline Pyramica schulzi & 0 & 0 & 0 & 2 & 0 & 3 \\
\hline Rogeria sp.1 & 0 & 1 & 1 & 0 & 0 & 0 \\
\hline Rogeria sp.4 & 1 & 1 & 1 & 3 & 0 & 3 \\
\hline Rogeria sp. gp. creightoni & 1 & (n) & (n) & 0 & 0 & 1 \\
\hline Sericomyrmex sp.1 & 12 & 5 & 15 & 16 & 10 & 17 \\
\hline Solenopsis sp.1 & 13 & 14 & 15 & 30 & 12 & 15 \\
\hline Solenopsis sp.2 & 1 & 0 & 6 & 0 & 1 & 11 \\
\hline Solenopsis sp.3 & 2 & 1 & 13 & 5 & 4 & 18 \\
\hline Solenopsis sp.4 & 0 & 0 & 1 & 0 & 0 & 0 \\
\hline Solenopsis substituta' & 0 & 2 & 0 & 1 & 1 & 0 \\
\hline Thaumatomyrmex mutilatus & 8 & 1 & 4 & 9 & 4 & 1 \\
\hline Trachymyrmex sp.1 & 5 & 2 & 2 & 2 & 2 & 4 \\
\hline Trachymyrmex sp.2 & 3 & (n) & 2 & 1 & 0 & 0 \\
\hline Trachymyrmex sp.3 & 17 & 12 & 6 & 18 & 7 & 10 \\
\hline Trachymyrmex sp.4 & 1 & 0 & 0 & 1 & 1 & 0 \\
\hline Trachymyrmex sp.5 & 1 & 0 & 0 & 0 & 0 & 0 \\
\hline Trachymyrmex sp.6 & 0 & 0 & 0 & 1 & 0 & 0 \\
\hline Wasmannia sp.1 & 0 & 0 & 1 & 0 & 0 & 0 \\
\hline Wasmannia auropunctata & 4 & 5 & 11 & 13 & 6 & 11 \\
\hline Total & 204 & 140 & 232 & 284 & 178 & 324 \\
\hline
\end{tabular}

R. Árvore, Viçosa-MG, v.28, n.1, p.139-146, 2004 
Portanto, o comportamento nas duas áreas selecionadas para o estudo pode ser explicado pela grande aptidão à rápida colonização e à fácil recuperação que as comunidades desses insetos possuem. Mackay et al. (1991) demonstraram esse fato em um trabalho sobre o impacto do fogo sobre a diversidade de formigas, concluindo que as espécies que colonizaram mais rapidamente foram as generalistas, pois possuem nichos mais amplos, podendo formar ninhos em muitos locais, além de utilizarem várias fontes alimentares. Fato similar a este pode ter ocorrido nas áreas em que foram realizadas as capinas. Outra hipótese é que, após a capina química, a vegetação morta e parte da viva se mantiveram presentes no local, facilitando talvez a recolonização das formigas, pois nem todo o sub-bosque foi eliminado, mas apenas as gramíneas e algumas plantas herbáceas, predominando no local os vegetais de folhas largas.

Thaumatomyrmex mutilatus, uma espécie de ambientes conservados e que merece destaque, por ser predador especializado em miriápodes (Delabie et al., 2000), também ocorreu nas áreas de eucaliptais antes da realização dos tratamentos (Quadro 1). Tal fato pode ser explicado pela presença de um sub-bosque nas áreas, o que provavelmente aumentou a variabilidade de locais disponíveis de forrageamento, nidificação e alimento, por exemplo.

Os resultados mostraram que apenas 11 espécies representadas nos dois talhões são coletadas pelo menos 20 vezes em cada um, além de Pheidole fallax e Pyramica eggersi, que tiveram uma freqüência bastante alta (44) e (42) no talhão onde houve a capina mecânica (Quadro 1). Analisando as respostas das 11 outras espécies (9 Myrmicinae, 1 Formicinae e 1 Ponerinae), constatouse que houve respostas diferentes em função do tipo de capina realizada. Foi encontrada também uma morfoespécie de Solenopsis, cuja freqüência aumentou bastante ao final dos 60 dias, em ambos os talhões.

Apenas duas espécies foram afetadas negativamente pela capina mecânica: Trachymyrmex sp.3 e Mycocepurus goeldii, enquanto quatro espécies foram afetadas pela capina química: Brachymyrmex sp.1, Solenopsis sp.1, Trachymyrmex sp.3 e Wasmannia auropunctata. Nota-se que apenas Trachyrmyrmex. sp.3 foi afetada em ambos os tratamentos.

Quadro 2 - Número médio de espécies de formigas por amostra em função do período, antes e depois da aplicação dos tratamentos, em eucaliptais. Bom Despacho-MG/Brasil. J unho a setembro de 2000. As médias seguidas pela mesma letra minúscula na linha e maiúscula na coluna não diferem entre si Tukey, p 0,05)

Table 2 - Average number of ant species per sample according the time before and after treatment application on Eucalyptus plantations. Bom Despacho, MG, Brazil. J une to September, 2000. Averages followed by the same lower-case letter in the line and upper-case letter in the column do not differ among them (Tukey, p 0.05)

\begin{tabular}{|l|c|c|c|c|c|}
\hline \multirow{2}{*}{ Tratamento } & \multicolumn{3}{|c|}{ Número de Espécies por Amostra } & \multicolumn{2}{c|}{ Data de Amostragem* } \\
\cline { 2 - 6 } & Antes do tratamento & 8 dias após & 60 dias após & 8 dias após & 60 dias após \\
\hline Capina mecânica & 4,1 & 2,8 & 4,7 & $-1,3 \mathrm{bB}$ & $-0,6 \mathrm{aA}$ \\
\hline Capina química & 5,7 & 3,6 & 6,4 & $-2,1 \mathrm{bB}$ & $-0,8 \mathrm{aA}$ \\
\hline
\end{tabular}

Quadro 3 - Número de espécies observadas e estimadas (Chao2), índice de diversidade de Shannon-Weaver (H) e porcentagem da riqueza inicial observada em função do período, antes e depois da aplicação dos tratamentos, em eucaliptais. Bom Despacho-M G/Brasil. J unho a setembro de 2000

Table 3 - Number of observed and estimated species (Chao2) and diversity index of Shannon-Weaver $(\mathrm{H})$ and \% of the observed initial richness as a function of time, before and after treatment application, in Eucalyptus plantations. Bom Despacho, MG, Brazil. J une to September 2000

\begin{tabular}{|c|c|c|c|c|c|c|c|c|c|}
\hline \multirow[b]{2}{*}{ Tratamento } & \multicolumn{3}{|c|}{8 Dias Antes } & \multicolumn{3}{|c|}{8 Dias Depois } & \multicolumn{3}{|c|}{60 Dias Depois } \\
\hline & $\begin{array}{c}\text { Riqueza } \\
\text { observada }\end{array}$ & $\begin{array}{l}\text { Riqueza } \\
\text { estimada } \\
\text { (Chao 2) }\end{array}$ & $H$ & $\begin{array}{c}\text { Riqueza } \\
\text { observada }\end{array}$ & $\begin{array}{c}\text { Riqueza } \\
\text { estimada } \\
\text { (Chao 2) }\end{array}$ & $H$ & $\begin{array}{c}\text { Riqueza } \\
\text { observada }\end{array}$ & $\begin{array}{l}\text { Riqueza } \\
\text { estimada } \\
\text { (Chao 2) }\end{array}$ & $H$ \\
\hline Capina mecânica & $\begin{array}{c}49 \\
(100 \%)\end{array}$ & 58,8 & 3,4 & $\begin{array}{c}39 \\
(79,5 \%)\end{array}$ & 49,9 & 3,2 & $\begin{array}{c}41 \\
(83,6 \%)\end{array}$ & 47,3 & 3,3 \\
\hline Capina química & $\begin{array}{c}57 \\
(100 \%)\end{array}$ & 73,1 & 3,6 & $\begin{array}{c}41 \\
(71,9 \%)\end{array}$ & 57,3 & 3,3 & $\begin{array}{c}48 \\
(84,2 \%)\end{array}$ & 72,5 & 3,4 \\
\hline
\end{tabular}


Outras seis espécies (Anochetus diegensis, Camponotus rufipes, Pheidole sp.9, Pheidole fallax, Pyramica eggersi e Sericomyrmex sp.1) foram mais freqüentes 60 dias após os tratamentos, notando-se que não houve nenhum efeito negativo sobre essas formigas em relação aos dois tratamentos. $\mathrm{O}$ incremento dessas seis espécies foi certamente devido a alterações no nicho ecológico. A capina, conseqüentemente, obrigou algumas dessas formigas a aumentar seu território de forrageamento ou diminuiu a competição com as demais espécies que usavam os mesmos recursos.

O gênero Anochetus não foi diretamente afetado pelas capinas no final do tratamento, provavelmente porque é um predador generalista de solo e serapilheira, não ocorrendo no extrato arbustivo presente no subbosque. C. rufipes é uma espécie que nidifica debaixo de troncos de árvores caídos ou debaixo de pedras; é bastante agressiva e territorialista, além de ter um poder de adaptação e colonização muito grande em habitat impactado (Caetano et al., 2002), o que mostra a sua capacidade de resiliência, ou seja, o retorno ao estado original após o distúrbio do local.

O mesmo ocorreu com o gênero Pheidole, que também não foi diretamente afetado, o que pode ser constatado quando se analisa o aumento da sua frequiência aos 60 dias das capinas. É importante destacar a espécie $P$. fallax, que segundo Marinho et al. (2002) apresentou alta frequiência em uma área de vegetação nativa de cerrado. Para os autores esse comportamento foi inesperado, pois essa formiga é característica de ambientes antropizados. Esse fato pode ser comprovado diante da alta frequiência da espécie no final das coletas. P. eggersi é uma espécie que se comportou da mesma forma diante dos tratamentos, talvez por ser predadora especialista de Collembola (Fowler, 1991). É provável que suas presas não tenham sido afetadas pelas capinas, tendo indiretamente favorecido o crescimento das suas colônias, havendo, então, um aumento considerável na frequiência da espécie.

Sericomyrmex é um gênero da tribo Attini que se utiliza de material vegetal vivo, ou não, para o cultivo de seu fungo e que, segundo a classificação de Silvestre (2000) das guildas de formigas do cerrado, é colocado como formigas cultivadoras de fungos a partir de matéria em decomposição. Marinho et al. (2002) constataram que esse gênero é comum em ambientes degradados, com ocorrência alta em áreas de eucaliptais, o que comprova o resultado desta pesquisa.

R. Árvore, Viçosa-MG, v.28, n.1, p.139-146, 2004
Constatou-se também que algumas espécies de formigas foram particularmente mais suscetíveis à capina com herbicida, como Brachymyrmex sp.1, Solenopsis sp.1 e W. auropunctata. Muitas espécies de Brachymyrmex e Solenopsis, e secundariamente de W. auropunctata, são formigas arborícolas (Delabie, 1988), o que pode ter levado à diminuição das frequiências diante da capina química. Segundo Delabie et al. (2000), as espécies que compõem esses gêneros forrageiam no solo, na serapilheira e também na vegetação, além de serem formigas bastante sensíveis às modificações do seu habitat. Um ponto de suma importância para o comportamento de W. auropunctata diante desse tratamento é a sua associação simbiótica com o Planococcus citri, uma espécie de Homoptera (Coccidea: Pseucoccidae) (Delabie et al., 1994).

M. goeldii foi sensível apenas em relação à capina mecânica. É uma espécie que alimenta seu fungo simbionte com restos de flores e frutas e com excrementos de pássaros, insetos e outros animais (Caetano et al., 2002). Provavelmente, a capina mecânica alterou o nicho ecológico relacionado com a manutenção dos substratos alimentares do fungo, o que explica, assim, a frequiência observada.

A espécie Trachymyrmex sp.3, por sua vez, foi afetada negativamente tanto na capina mecânica, quanto na química. Segundo Silvestre (2000), esta é uma Attini desfolhadora. Observou-se a queda da sua frequiência no final do tratamento, decorrida provavelmente da retirada da vegetação de sub-bosque, onde certamente essas formigas forrageavam normalmente.

Conclui-se que qualquer tipo de capina perturba a comunidade de formigas momentaneamente, apresentando um efeito deletério imediato, de igual intensidade e de pouca duração sobre a diversidade de Formicidae. Apesar disto, sabe-se que parte da diversidade é afetada principalmente pela capina mecânica, em que foi constatada maior perturbação da comunidade. Muitos efeitos negativos foram atribuídos à diminuição da disponibilidade dos recursos alimentares diretamente utilizáveis, assim como dos organismos que vivem associados com numerosas espécies de formigas (Delabie, 2001).

Deve-se então recomendar, quando este tipo de manejo se fizer indispensável, o uso da capina química, por ser a prática que causa menos efeitos negativos à 
comunidade de formigas (e certamente à fauna em geral). No entanto, é preciso iniciar uma intensa reflexão no meio científico, de um lado sobre o relacionamento que há entre fauna e flora (com a manutenção ecologicamente correta e desejável de sua biodiversidade) e de outro sobre a importância do crescimento do sub-bosque nativo em meio às plantações de eucalipto.

\section{AGRADECIMENTOS}

À CAPES e à Empresa CAF Santa Bárbara Ltda., pelo suporte financeiro. Ao CEPEC/CEPLAC (Lucileide de Souza Ramos e Mércia Dayane de Santana Maia), pelo auxílio nas montagens dos formicídeos; e aos funcionários da Empresa CAF, João Batista Rodrigues e Geraldo Gonçalves Pereira, pelo auxílio nas coletas.

\section{REFERÊNCIAS BIBLIOGRÁFICAS}

ALMEIDA, A. F.; ALVES, J. E. M.; MENDES FILHO, J. M.A. Manutenção de sub-bosque em florestas de Eucalyptus urophylla e a distribuição regular de porta-iscas, visando o controle preventivo de saúvas (Atta spp.). Silvicultura, v. 8, n. 28 , p. $142-144,1983$ a.

ALMEIDA, A. F. et al. A avifauna e o sub-bosque como fatores auxiliares no controle biológico das saúvas em florestas implantadas. Silvicultura, v. 8, n. 28, p. 145-150, 1983 b.

ANDERSEN, A. N. et al. Using ants as bioindicators in land management: simplifying assessment of ant community responses. Journal of Applied Ecology. v. 39, p. 8-17, 2002.

BESTELMEYER, B. T. et al. Soil microarthropods of a rubber plantation and a natural forest. Environment \& Ecology, v. 3, n. 2, p. 143-147, 2000.

BOLTON, B. A new general catalogue of the ants of the world. Cambridge, MA: Harvard University Press, 1995. $504 \mathrm{p}$.

CAETANO, F. H.; JAFFÉ, K.; ZARA, F. J. Formigas: biologia e anatomia. São Paulo: Topázio, 2002. 131 p.

COLWELL, R. K. EstimateS: Statistical estimation of species richness and shared species from samples. Version $5.0 \mathrm{bl}$ User's Guide and application published. University of Connecticut, USA, 1997. Disponível em: http:// viceroy.eeb.uconn.edu/estimates.

DELABIE, J. H. C. Ocorrência de Wasmannia auropunctata (Hymenoptera, Formicidae, Myrmicinae) em cacauais na Bahia. Revista Theobroma, v. 18, n. 1, p. 2937, 1988.
DELABIE, J. H. C.; ENCARNAÇÃO, A. M. V.; CARZOLA, I. M. Relations between the little fire ant, Wasmannia auropunctata, and its associated mealybug, Planococcus citri, in Brazilian cocoa farms, p, 91-103. In: WILLIAMS, D. F. (Ed) Exotic Ants: biology, impact, and control of introduced species. Boulder: Westview Press, 1994. $332 \mathrm{p}$.

DELABIE, J. H. C. Comunidades de formigas (Hymenoptera. Formicidae) métodos de estudo e estudos de casos na Mata Atlântica. In: ENCONTRO DE ZOOLOGIA DO NORDESTE, 1999, Feira de Santana-BA. Anais... Feira de Santana: 1999. p. 58-68.

DELABIE, J. H. C. et al. Litter and soil ant communities: how many samples need to be taken. In: AGOSTI, D. et al. Ants: standart methods for measuring and monitoring biodiversity. Washington: Smithsonian Institution, 2000. 280 p.

DELABIE, J. H. C. Trophobiosis between Formicidae and Hemiptera (Sternorrhyncha and Auchenorrhyncha): an overview. Neotropical Entomology, v. 30, n. 4, p. 501-516, 2001.

FARIA, F. S. B. et al. Uso da armadilha "pit-fall" na avaliação dos efeitos da fragmentação florestal em comunidades de formigas epígeas da mata atlântica. In: ENCONTRO DE MIRMECOLOGIA, 15., 2001, Londrina. Anais... Londrina: 2001. p. 365-368.

FOWLER, H. G. et al. Ecologia Nutricional de formigas. In: PANIZZI, A. R.; PARRA, J. R. P. (Eds). Ecologia Nutricional de Insetos e suas Implicações no Manejo de Pragas. São Paulo: Manole e CNPq, 1991. 359 p.

GOLFARI, L. Zoneamento ecológico do Estado de Minas Gerais para reflorestamento. Belo Horizonte: Centro de Pesquisa Florestais da Região de Cerrado, 1975. 65 p. (Série Técnica, 3).

LIMA, W. P. Impacto ambiental do eucalipto. São Paulo: EDUSP, 1993. $301 \mathrm{p}$.

LOUZADA, J. N. C.; SANCHES, N. M.; SCHILINDWEIN, M. N. Bioindicadores de qualidade e de impactos ambientais da atividade agropecuária. Informe Agropecuário, v. 21, n. 202, p. 72-77, 2000.

MACKAY, W. P. et al. Impact of slashing and burning of a tropical rain forest on the native ant fauna (Hymenoptera: Formicidae). Sociobiology, v. 18, n. 3, p. 257-268, 1991.

MARINHO, C. G. S. et al. Diversidade de formigas (Hymenoptera: Formicidae) da serapilheira em eucaliptais (Myrtaceae) e área de cerrado de Minas Gerais.

Neotropical Entomology, v. 31, n. 2, p. 187-195, 2002.

R. Árvore, Viçosa-MG, v.28, n.1, p.139-146, 2004 
PAULA, J. A. Biodiversidade, população e economia: uma região de Mata Atlântica. Belo Horizonte: Universidade Federal de Minas Gerais, 1997. 672 p.

PECK, S. L.; MCQUAID, B.; CAMPBELL, L. Using ant species (Hymenoptera: Formicidae) as a biological indicator of agroecossystem condition. Environmental Entomology. v. 27, n. 5 , p. 1102-1110, 1998.
RAMOS, L. S. et al. Impacto de iscas formicidas granuladas sobre a mirmecofauna não-alvo em eucaliptais segundo duas formas de aplicação. Neotropical Entomology, v. 32, n. 2, p. 231-237, 2003.

SILVESTRE, R. Estrutura de comunidades de formigas do cerrado. 2000. $216 \mathrm{f}$. Tese (Doutorado em

Entomologia) - Universidade Estadual de São Paulo, Ribeirão Preto, 2000. 